\title{
A NOTE ON A GLOBAL STABILITY THEOREM OF M. W. HIRSCH
}

\author{
JIANG JI-FA
}

(Communicated by Kenneth R. Meyer)

\begin{abstract}
For a 3-dimensional cooperative vector field $F$, we present a new global stability result which is the same as that of Hirsch [4] except that we do not assume that $\operatorname{Div} F \leq 0$ or that the equilibrium is stable. Thus we solve the "interesting problem" pointed out by Hirsch [4].
\end{abstract}

\section{INTRODUCTION}

In a series of papers [1-4], Hirsch investigated cooperative systems and obtained many very important results, which show that most trajectories are stable and approach the set of equilibria and that limit sets are invariant sets of systems with dimension one lower. Using the order-preserving properties of cooperative systems and the first Čech cohomology group, he [4] proved that for 3-dimensional cooperative systems every cycle $K$ lies on the boundary of a semi-invariant closed 3-cell which is contained in every order interval containing $K$. Applying this result, he showed the following global stability theorem:

Theorem A (Hirsch). Let $F: X \rightarrow \mathbb{R}^{3}$ be a $C^{1}$ cooperative vector field. Suppose the following conditions hold:

(a) $X=\mathbb{R}^{3}$, or Int $\mathbb{R}_{+}^{3}$, or $[[p, q]]$;

(b) $\operatorname{Div} F<0$ in a dense set;

(c) every forward semi-orbit has compact closure in $X$; and

(d) there is a unique equilibrium $p$, and it is asymptotically stable.

Then $p$ is globally asymptotically stable.

Meanwhile, he pointed out that "It is an interesting problem to determine the validity of Theorem A (for cooperative systems) when hypothesis (b) is weakened to $\operatorname{Div} F \leq 0$, or when stability of $p$ is not assumed." For irreducible cooperative systems he proved that the conclusion of Theorem A holds under considerably weaker hypotheses: In addition to (a) and (c), he need only assume

(e) there is not more than one equilibrium.

It is the purpose of this note to prove the following theorem.

Received by the editors January 16, 1990.

1980 Mathematics Subject Classification (1985 Revision). Primary 34D05, 34D20.

Key words and phrases. Cooperative systems, global stability. 
Theorem B. If $F: X \rightarrow \mathbb{R}^{3}$ is a $C^{\prime}$ cooperative vector field and the conditions (a), (c), and (e) hold, then there exists a unique equilibrium $p$ and it is globally asymptotically stable.

Our result shows that, in order to guarantee that $F$ has a globally asymptotically stable equilibrium, the hypotheses (b), stability of $p$, and irreducibility are not necessary. Therefore, we solve the "interesting problem" pointed out by Hirsch.

Remark. Obviously, (c) and (e) are necessary conditions for $F$ to have a globally asymptotically stable equilibrium.

\section{Proof of TheOREM B}

We first establish some definitions and notation.

A $C^{1}$ vector field $F: X \rightarrow \mathbb{R}^{n}$ is called cooperative if its derivative matrices $D F(x)$ have nonnegative off-diagonal entries.

We use the following notation for the vector order in $\mathbb{R}^{3}$ :

$$
\begin{aligned}
& x \leq y \quad \text { if } x^{i} \leq y^{i} \quad(i=1,2,3), \\
& x<y \quad \text { if } x \leq y \quad \text { and } x \neq y, \\
& x \ll y \quad \text { if } x^{i}<y^{i} \quad(i=1,2,3) .
\end{aligned}
$$

Notations such as $y \gg x$ have the natural meanings.

For any points $u, v$ in $\mathbb{R}^{3}$, define the closed order interval $[u, v]=\{x$ : $u \leq x \leq v\}$ and the open order interval $[[u, v]]=\{x: u \ll x \ll v\}$.

The interior and the boundary of a set $S$ are denoted by $\operatorname{Int}(S)$ and $\partial S$, respectively. The flow generated by $F$ is denoted by $\phi_{t}(x)=x(t)$. Let $\omega(x)$ denote the omega limit set of $x \in X$ and $\mathbb{R}_{+}^{3}=\left\{x \in \mathbb{R}^{3}: x \geq 0\right\}$. In the following, we consider only 3 -dimensional cooperative systems.

Lemma 1. For $x \in X$ there cannot exist $u, v$ in $\omega(x)$ with $u \ll v$.

Lemma 2. Suppose $[a, b] \subset X, a \ll b$, and $[a, b]$ contains a cycle $K$. Then $[a, b]$ contains an equilibrium $p$ which is unrelated by $\ll$ or $\gg$ to any point of $K$.

Lemmas 1 and 2 and their proofs can be found in [4, p. 96 and p. 99].

Lemma 3. If the hypotheses (a), (c), and (e) hold, then $F$ has a unique equilibrium $p$.

Proof. Fix $x \in X$. If $\omega(x)$ contains an equilibrium, then the conclusion follows from (e). If $\omega(x)$ contains no equilibrium, then $\omega(x)$ is a cycle [1, Theorem 4.1]. Therefore, the conclusion follows from Lemma 2 and (e).

Lemma 4. Let $F$ be a 2-dimensional cooperative vector field and $x(t)=\left(x^{1}(t)\right.$, $\left.x^{2}(t)\right)$ be a solution defined for $-\infty<t \leq 0$, or for $0 \leq t<\infty$. Then $x^{i}(t)$ is monotone for $|t|$ sufficiently large.

This lemma is adapted from [2, p. 432]. 
Set $\pi^{i}=\left\{x \in \mathbb{R}_{+}^{3}: x^{i}=0\right\}$. Recall from Lemma 3 that the system has a unique equilibrium at $p=\left(p^{1}, p^{2}, p^{3}\right)$.

Lemma 5. Suppose $\omega(x) \subset p+\partial \mathbb{R}_{+}^{3}$, where the operation + denotes setwise addition. If $y=\left(y^{1}, y^{2}, y^{3}\right) \in \omega(x)$ with $y^{i}>p^{i}$ and $y^{j}>p^{j}$ for distinct $i, j, k$, then $y(t) \in p+\pi^{k}$ for $t \in \mathbb{R}$.

Proof. We prove the case $i=1, j=2, k=3$. Proofs of other cases can be carried out in a similar manner.

By invariance, $y(t) \in \omega(x) \subset p+\partial \mathbb{R}_{+}^{3}$ for $t \in \mathbb{R}$. It follows from $y \in p+\partial \mathbb{R}_{+}^{3}$ and $y^{i}>p^{i}$ for $i=1,2$ that $y^{3}=p^{3}$. Therefore, $y(t) \in p+\operatorname{Int}\left(\pi^{3}\right)$ for $|t|$ sufficiently small. Set

$$
t^{*}=\sup \left\{t: y^{3}(\tau)=p^{3} \text { for all } \tau \in[0, t]\right\} .
$$

Obviously, $t^{*}>0$. We assert that $t^{*}=+\infty$. Otherwise, $t^{*}<+\infty$. By the definition of $t^{*}$ and the continuity of $y(t), y^{3}(t)=p^{3}$ for $t \in\left[0, t^{*}\right]$, and either $y^{1}\left(t^{*}\right)=p^{1}$ or $y^{2}\left(t^{*}\right)=p^{2}$. Without loss of generality, we suppose the latter case occurs. Let $F=\left(F_{1}, F_{2}, F_{3}\right)$. Then $\left(y^{1}(t), y^{2}(t)\right)$ for $t \in\left[0, t^{*}\right]$ is a solution of the following 2-dimensional cooperative system:

$$
\begin{aligned}
& \dot{x}=F_{1}\left(x, y, p^{3}\right) \\
& \dot{y}=F_{2}\left(x, y, p^{3}\right) .
\end{aligned}
$$

It is easy to see that $(x, y)=\left(p^{1}, p^{2}\right)$ is also a solution of (1). It follows from $p^{1}<y^{1}, p^{2}<y^{2}$, and the Kamke theorem [5, p. 27] that $p^{1}<y^{1}\left(t^{*}\right)$ and $p^{2}<y^{2}\left(t^{*}\right)$, contradicting $y^{2}\left(t^{*}\right)=p^{2}$. This contradiction shows that $t^{*}=+\infty$; that is, $y(t) \in p+\pi^{3}$ for $t \geq 0$. If there is $t_{1}<0$ such that $y\left(t_{1}\right) \notin$ $p+\pi^{3}$, then $y\left(t_{1}\right) \in\left(p+\operatorname{Int}\left(\pi^{i}\right)\right)$ for $i \neq 3$. Suppose $y\left(t_{1}\right) \in p+\operatorname{Int}\left(\pi^{2}\right)$. Then, as just proved, $y(t) \in p+\pi^{2}$ for $t \geq t_{1}$, which contradicts $y \in p+\operatorname{Int}\left(\pi^{3}\right)$. This contradiction proves Lemma 5 .

Proof of Theorem B. We first show that $\omega(x)=p$ for $x \in\left(p+\mathbb{R}_{+}^{3}\right) \cap X$. Observe that $\omega(x) \subset\left(p+\mathbb{R}_{+}^{3}\right) \cup X$, by monotonicity of the flow. Suppose first that $\omega(x)$ contains no equilibrium. Then $\omega(x)$ is a cycle (see [1, Theorem 4.1]). By Lemma $2,\left(p+\mathbb{R}_{+}^{3}\right) \cap X$ contains an equilibrium $q$ which is unrelated by $\ll$ or $\gg$ to any point of $\omega(x)$. By Lemma 3, $q=p$. Therefore, $\omega(x) \subset p+\partial \mathbb{R}_{+}^{3}$. Because $\omega(x)$ is a cycle, there is a point $y=\left(y^{1}, y^{2}, y^{3}\right) \in \omega(x)$ such that $y^{i}-p^{i}>0$ for two indices $i$. By Lemma $5, \omega(x) \subset p+\pi^{j}$ for some $j$, that is, $\omega(x)$ is a cycle of a 2-dimensional cooperative system, contradicting Lemma 4. This contradiction shows that $\omega(x)$ contains the equilibrium $p$. If $\omega(x)-p \neq \varnothing$, then, by the uniqueness of the equilibrium $p$, there exists a point $y \in \omega(x)$ such that $y^{i}-p^{i}>0$ for two indices $i$. By Lemma 5 , $y(t) \in p+\pi^{j}$ for some $j$ and all $t \in \mathbb{R}$; that is, $y(t)$ is a solution of a 2dimensional cooperative system. Restricting attention to that 2-dimensional 
system, and applying Lemmas 3 and 4, we know that $\lim t \rightarrow \pm \infty y(t)=p$. Therefore, there exists a real number $T>0$ such that $y(-T)<y$. By the Kamke theorem,

$$
p<y(-T)<y<y(T)<\cdots<y(n T) .
$$

It follows that $p=\lim n \rightarrow \infty y(n T) \geq y>p$, a contradiction. This proves that $\omega(x)=p$ for $x \in\left(p+\mathbb{R}_{+}^{3}\right) \cap X$. In an analogous way, we can prove that $\omega(x)=p$ for $x \in\left(p-\mathbb{R}_{+}^{3}\right) \cap X$. Hence, for any $x \in X$, there are points $u \ll x \ll v$ such that the forward orbits of $u$ and $v$ tend to $p$. The Kamke theorem now shows that the forward orbit of $x$ also limits at $p$; i.e., $\lim t \rightarrow \infty x(t)=p$ for all $x \in X$.

It remains to prove that $p$ is stable. For any neighborhood $U \subset X$ of $p$, we can choose $u, v$ in $X$ such that $u \ll p \ll v$ and $[u, v] \subset U$. Since $\lim t \rightarrow \infty u(t)=\lim t \rightarrow \infty v(t)=p$, there is a $T>0$ such that $u(t), v(t) \in$ $[u, v]$ for $t \geq T$. Set $V=[[u(T), v(T)]]$. Then for any $x \in V$ we have $u(t+T) \ll x(t) \ll v(t+T)$ for $t \geq 0$. Therefore,

$$
x(t) \in[[u(t+T), v(t+T)]] \subset[u, v] \subset U ;
$$

i.e., $\phi_{t}(V) \subset U$ for $t \geq 0$. By the definition of Liapunov stability, $p$ is stable. This completes the proof of Theorem B.

\section{ACKNOWLEDGMENT}

The author would like to thank the referee for valuable suggestions.

\section{REFERENCES}

1. M. W. Hirsch, Systems of differential equations that are competitive or cooperative. I: Limit sets, SIAM J. Math. Anal. 13 (1982), 167-179.

2. __ Systems of differential equations that are competitive or cooperative. II: Convergence almost everywhere, SIAM J. Math. Anal. 16 (1985), 432-439.

3. __ Systems of differential equations that are competitive or cooperative. III: Competing species, Nonlinearity 1 (1988), 51-71.

4. __ Systems of differential equations that are competitive or cooperative. V: Convergence in 3-dimensional systems, J. Differential Equations 80 (1989), 94-106.

5. W. A. Coppel, Stability and asymptotic behavior of differential equations, D. C. Heath, Lexington, 1965. 